University of Nebraska - Lincoln

DigitalCommons@University of Nebraska - Lincoln

Faculty Publications - Textiles, Merchandising and Fashion Design

Textiles, Merchandising and Fashion Design,

Department of

2012

\title{
Investigation of the Structure and Properties of Silk Fibers Produced by Actias lunas
}

Narendra Reddy

University of Nebraska - Lincoln, nreddy3@unl.edu

Yiqi Yang

University of Nebraska-Lincoln, yyang2@unl.edu

Follow this and additional works at: https://digitalcommons.unl.edu/textiles_facpub

Reddy, Narendra and Yang, Yiqi, "Investigation of the Structure and Properties of Silk Fibers Produced by Actias lunas" (2012). Faculty Publications - Textiles, Merchandising and Fashion Design. 33.

https://digitalcommons.unl.edu/textiles_facpub/33

This Article is brought to you for free and open access by the Textiles, Merchandising and Fashion Design, Department of at DigitalCommons@University of Nebraska - Lincoln. It has been accepted for inclusion in Faculty Publications - Textiles, Merchandising and Fashion Design by an authorized administrator of DigitalCommons@University of Nebraska - Lincoln. 


\title{
Investigation of the Structure and Properties of Silk Fibers Produced by Actias lunas
}

\author{
Narendra Reddy ${ }^{1}$ and Yiqi Yang1, 2,3 \\ 1. Department of Textiles, Merchandising and Fashion Design, University of Nebraska- Lincoln, \\ 234 HECO Building, East Campus, Lincoln, NE 68583-0802, USA \\ 2. Department of Biological Systems Engineering, University of Nebraska- Lincoln, \\ 234 HECO Building, East Campus, Lincoln, NE 68583-0802, USA \\ 3. Nebraska Center for Materials and Nanoscience, University of Nebraska- Lincoln, \\ 234 HECO Building, East Campus, Lincoln, NE 68583-0802, USA \\ Corresponding author - Yiqi Yang, email yyang2@unl.edu
}

\begin{abstract}
This paper reports the structure and properties of silk fibers produced by Actias lunas in comparison to Bombyx mori and the common wild silks. Considerable efforts are being made to find new sources for natural silk and also to develop regenerated protein fibers to supplement the limited amounts of B. mori and wild silks available in the market. In addition, it has been found that non-traditional silks have unique properties and utilizing uncommon wild silks can provide income and employment to indigenous people where the wild silks are found. Actias lunas belongs to the Saturniidae family of silk producing insects. However, the structure and properties of silk produced by A. lunas have not been studied. This research showed that the silk fibers produced by the luna moth had morphological and physical structure similar to that of the common wild silks but tensile properties similar to that of B. mori silk. A. lunas silk fibers are composed of higher amounts of hydrophobic amino acids and had much less glycine than B. mori and common wild silks. With a fineness of 2 denier, breaking tenacity of $4.3 \mathrm{~g} /$ den and breaking elongation of $10.9 \%$, the tensile properties of $A$. lunas silk fibers were similar to that of $B$. mori and much better than that of the common wild silks that are coarser and have lower breaking tenacity. A. lunas fibers show good potential to be useful for applications currently using $B$. mori silk.
\end{abstract}

Keywords: Actias lunas, silk fibers, wild silks, properties

\section{Introduction}

Bombyx mori and the wild silks Antheraea mylitta, Antheraea pernyi, and Phylisomia ricini belonging to the Saturniidae family are the most common types of silks that have been known for centuries. Although silks are preferred fibers for various applications, there is relatively limited quantity of silk available compared to other fibers. The total annual world production of silk is approximately 1.3 million tons compared to 63 million tons of fibers consumed in 2009 [1]. Therefore, attempts have been made to find alternative sources for silk fibers. Such attempts have also led to the discovery of new sources for natural silk fibers and the development of regenerated protein fibers. Recently, we have demonstrated that high quality regenerated protein fibers with properties suitable for textile and medical applications can be produced from wheat gluten and soyproteins [2-4].

Although B. mori and the common wild silks are predominantly used for commercial scale silk production, many other insects have been known to produce silk and silk containing cocoons [5-8]. Efforts are being made to harvest the silks produced by the uncommon wild insects, study their properties and understand the potential of using the silks for various applications. Interests in non-traditional silks are mainly due to the po- 
tential for high income generation for indigenous people located in habitats where the wild silks are found [9, 10]. In addition, wild silks may have unique properties not found in the common silks. For instance, it has been reported that weaver ants produce silk in the form of nanofibers unlike any other known silk [11]. Similarly, silks produced by bees have a unique coiled-coiled structure compared to the normal silks. Recent studies have also shown that wild silks have better potential for cell growth than B. mori silk [12].

The Saturniidae family consists of some of the largest silk producing insects [5]. However, only a limited number of Saturniidae insects primarily A. mylitta, A. pernyi and $P$. ricini are currently used for commercial scale silk production. We have recently reported that several uncommon Saturniidae insects produce silk with properties similar or better than that of the common B. mori silks $[13,14]$. It was found that silk fibers produced by Coscinocera hercules had properties very similar to that of B. mori silk whereas Copaxa multifenestrata produced coarse silk fibers that had considerably lower tensile properties than B. mori and the common wild silks [13].

Actias lunas also belongs to the Saturniidae family but the properties of silk produced by the luna moths have not been studied. Luna moths are generally found in forested areas but are nocturnal and have a wing span ranging from 70 to $105 \mathrm{~mm}$. Depending on their location, Luna moths are reported to have one to three generations (univoltine or multivoltine). Reared Luna cocoons are reported to have a different color than those found in nature [15].

In this research, the cocoons produced by Luna moths were used to extract silk fibers and the structure and properties of the fibers have been studied in an effort to understand the unique properties and identify potential applications. The properties of the A. lunas silk have been compared to the properties of $B$. mori and the common wild silks reported in literature.

\section{Experimental}

\section{Materials}

A. lunas cocoons were obtained from Reiman Gardens in Ames, Iowa. Ethylenediamine and sodium carbonate used for degumming were analytical grade chemicals purchased from VWR international (Bristol, CT).

\section{Degumming}

The A. lunas cocoons were degummed by treating in a solution containing $0.5 \%(\mathrm{w} / \mathrm{w})$ sodium carbonate and $10 \%(\mathrm{w} / \mathrm{w})$ ethylenediamine solution at $95{ }^{\circ} \mathrm{C}$ for
30 min with a cocoon to solution ratio of 1:20. The fibers obtained after treatment were washed several times in warm water and dried under ambient conditions.

\section{Composition}

The composition of the A. lunas fibers was determined in terms of the amino acid content. Silk fibers were hydrolyzed using $6 \mathrm{~N} \mathrm{HCl}$ at $110{ }^{\circ} \mathrm{C}$ for $20 \mathrm{~h}$ under argon atmosphere. After the hydrolysis, the samples were evaporated to dryness and then redissolved in $200 \mu 1$ of $0.02 \mathrm{~N} \mathrm{HCl}$. Fifty microliters of the solution was injected onto the Hitachi (L-8800A) amino acid analyzer to determine the amino acid type and content. Norleucine was used as the internal standard and corrections were made to account for the dilutional errors.

\section{Physical Structure}

X-ray diffraction (Rigaku D-max/B $\Theta / 2 \Theta$ X-Ray diffractometer, Rigaku Americas, Woodlands, TX) was used to study the physical structure of the silk fibers in terms of the degree of crystallinity and shape and intensity of the diffraction peaks. X-ray diffraction measurements were taken using a Bragg-Brentano parafocusing geometry, a diffracted beam monochromator, and a copper target X-ray tube set to $40 \mathrm{kV}$ and $30 \mathrm{~mA}$. The silk fibers were powdered in a Wiley mill and the powder was pressed to form a pellet. The pellet was fixed to the sample holder and diffraction intensities were recorded from $5^{\circ}$ to $40^{\circ}(2 \theta)$. The degree of crystallinity (\%) of the fiber was obtained by integrating the area under the crystalline peaks after subtracting the background and air scatter using the program MICROCAL ORIGIN.

\section{Morphology}

Morphology of the undegummed A. lunas cocoons and fibers obtained after degumming were observed in a Hitachi S-3000N variable pressure scanning electron microscope (SEM). Longitudinal and cross-sectional images of the fibers were taken on samples mounted on conductive adhesive tapes and sputter coated with gold palladium.

\section{Tensile Properties and Moisture Regain}

Silk fibers obtained after degumming were tested for their tensile properties in terms of breaking strength, breaking elongation, and Young's modulus. Fibers were conditioned under the standard testing conditions of $21^{\circ} \mathrm{C}$ and $65 \%$ relative humidity for at least $24 \mathrm{~h}$ before conducting the tensile tests. Fineness of the fibers was measured in terms of denier (weight in grams per 9,000 
meters) by weighing a known length of fibers. Tensile tests were performed on an Instron (Model 4444) tensile testing machine using a gauge length of 1 inch and cross head speed of $18 \mathrm{~mm} / \mathrm{min}$. At least 50 fibers were tested to determine the tensile properties and the average and \pm one standard deviations are reported. The moisture regain of the fibers was determined according to ASTM standard method 2654 using standard conditions of $21^{\circ} \mathrm{C}$ and $65 \%$ relative humidity.

\section{Results and Discussion}

Properties of A. lunas Cocoons

Cocoons produced by A. lunas were white to slightly yellow in color and were extensively covered with leafy materials as seen from Fig. 1. The cocoons had a considerably uniform length of about $4 \mathrm{~cm}$ whereas the width of the cocoons ranged from 2 to $2.6 \mathrm{~cm}$. The cocoons used in this study had an average weight of $200 \mathrm{mg}$ and were considerably smaller compared to $B$. mori cocoons that had an average weight of approximately $640 \mathrm{mg}$ $[14,16]$. The wild silks $A$. mylitta and $P$. ricini cocoons are reported to produce cocoons with average weight of $3.4 \mathrm{~g}$ and $840 \mathrm{mg}$, respectively [17, 18]. However, cocoons as heavy at 14 grams have also been reported for A. mylitta cocoons [16]. The average thickness of the $A$. lunas cocoons was about $420 \mu \mathrm{m}$ whereas B. mori cocoons are reported to have thickness ranging from $500 \mu \mathrm{m}$ to $5 \mathrm{~mm}$. Fibers in the cocoons were relatively loosely arranged and could be pulled out easily whereas fibers in cocoons produced by some Saturniidae insects such as cecropia are tightly held by the cocoons and cannot be pulled out without degumming [14]. The A. lunas cocoons consisted of a single layer of fibers whereas most wild silk cocoons belonging to the saturniidae family are reported to have three or more distinct layers $[5,14]$.

Figure 2 shows the SEM picture of the undegummed Luna cocoon revealing the morphological features. Luna moth spins two fibers simultaneously, commonly observed in most silk producing insects. The fibers were long and run in random directions and form a network structure providing strength to the cocoon. Considerable amounts of white particles were seen on the fibers reported to be calcium oxalate crystals excreted by the silk worms [19, 20].

\section{Composition of Fibers}

Table 1 provides the amino acid composition of the $A$. lunas silk fibers in comparison to B. mori and wild silks. As seen from the table, the amino acids alanine, glycine, serine and tyrosine account for $66 \%$ of the amino acids in A. lunas silk fibers whereas these four amino acids

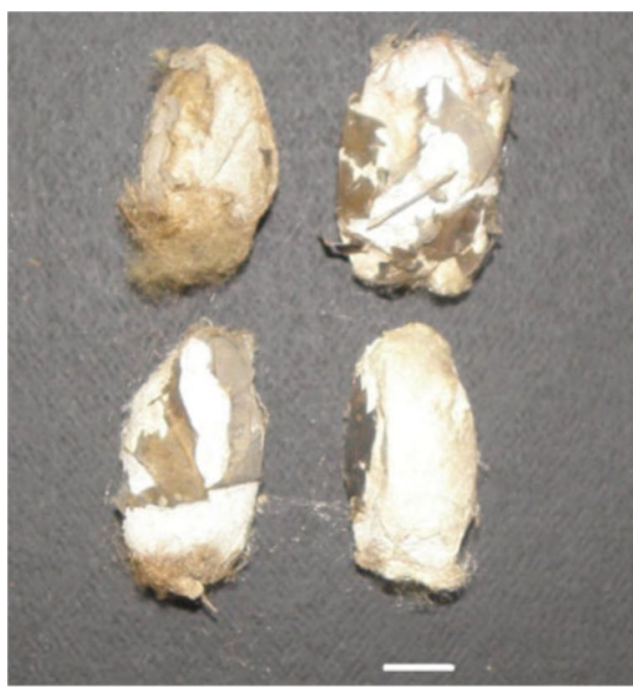

Fig. 1. Digital image of the A. lunas cocoons. Scale bar is $1 \mathrm{~cm}$.

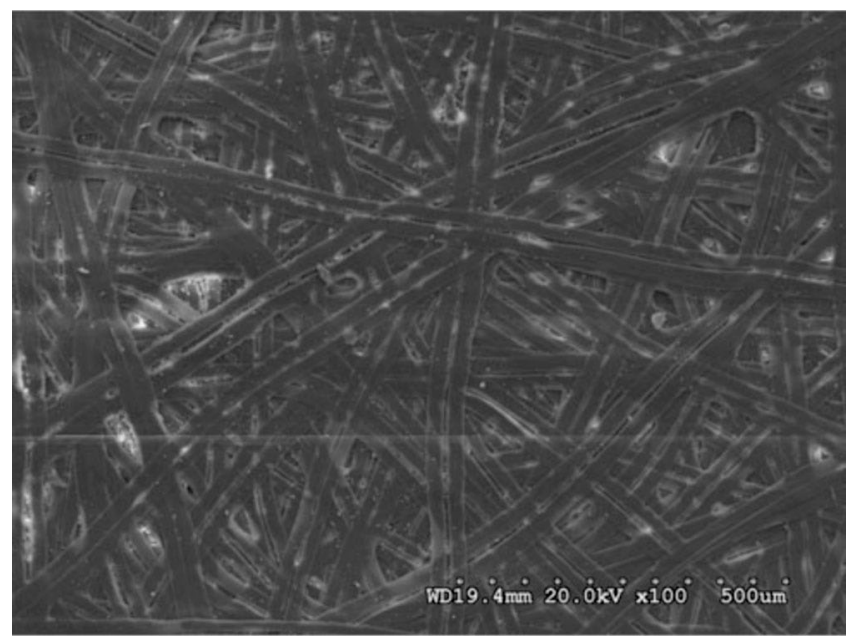

Fig. 2. SEM image of the surface of an undegummed $A$. lunas cocoon.

account for $90 \%$ in B. mori and approximately $78 \%$ in the wild silks $[17,18]$. The amino acids alanine, glycine, serine and threonine that are reported to be found in the crystalline region account for $53 \%$ of the amino acids in A. lunas silk whereas the amount of crystalline amino acids in B. mori silk is about $86 \%$ and about $72 \%$ in the wild silks. The ratio of amino acids in the crystalline and amorphous regions is called the disorder ratio and is reported to affect the structure and properties of the fibers. A. lunas silk had lower amounts of crystalline amino acids suggesting that the fibers may have poor mechanical properties compared to B. mori silks assuming that all other factors are the same. A. lunas silk had much higher amounts of aspartic acid, arginine, glutamic acid, leucine and threonine but very low levels of glycine compared to the common silks.

Table 2 shows the ratio of amino acids in A. lunas and the common silks. A. lunas silk had similar ratio of basic 
Table 1. Amino acid composition of A. lunas silk compared to B. mori and three varieties of common wild silks

\begin{tabular}{llllll}
\hline \multirow{2}{*}{$\begin{array}{l}\text { Amino } \\
\text { acids }\end{array}$} & \% Amino acids & & & \\
\cline { 2 - 6 } & A. lunas & B. mori & A. mylitta & A. pernyi & P. ricini \\
\hline Alanine & 22.3 & 28.4 & 34.1 & 34.7 & 36.3 \\
Glycine & 11.9 & 44.6 & 27.6 & 28.4 & 29.4 \\
Serine & 16.6 & 12.1 & 9.9 & 9.1 & 8.9 \\
Tyrosine & 15.3 & 5.2 & 6.8 & 5.1 & 5.8 \\
Aspartic acid & 6.6 & 1.3 & 6.1 & 5.0 & 3.9 \\
Arginine & 6.6 & 0.5 & 5.0 & 4.7 & 4.1 \\
Glutamic acid & 4.3 & 1.8 & 1.4 & 1.4 & 1.3 \\
Leucine & 7.5 & 0.7 & 0.7 & 0.7 & 0.7 \\
Threonine & 2.8 & 0.9 & 0.3 & 0.2 & 0.2 \\
Histidine & 0.7 & 0.1 & 0.8 & 0.7 & 0.8 \\
Lysine & 1.37 & 0.2 & 0.2 & 0.2 & 0.2 \\
Valine & 2.2 & 2.4 & 1.7 & 1.5 & 1.3 \\
Isoleucine & 0.9 & 0.8 & 0.6 & 0.5 & 0.4 \\
\hline
\end{tabular}

Data for B. mori and wild silks are from references [17, 18, 21, 22].

Table 2. Comparison of the amino acid ratios in A. lunas fibers to the common silks

\begin{tabular}{lllll}
\hline Ratio & A. lunas & B. mori & A. mylitta & P. ricini \\
\hline Basic/acidic & 0.70 & 0.65 & 0.97 & 1.30 \\
Hydrophilic/hydrophobic & 0.91 & 0.28 & 0.44 & 0.35 \\
Glycine/alanine & 0.55 & 1.58 & 0.81 & 0.80 \\
\hline
\end{tabular}

Data for B. mori and wild silks are from reference [17].

to acidic amino acids compared to B. mori but a much higher ratio of hydrophilic to hydrophobic amino acids suggesting a more uniform distribution of hydrophilic to hydrophobic amino acids in A. lunas silk. Based on the amino acid composition, A. lunas silk could have lower moisture sorption than the common silks.

\section{Morphology}

Silk fibers produced by A. lunas are flat and ribbonlike but had twists as seen from Fig. 3. Considerable amounts of white particles were seen on the fibers even after degumming. The white particles are reported to be calcium oxalate crystals secreted by the moths and are also found in other Saturniidae silks. Except for the presence of the crystals, the fibers had a smooth appearance as seen from Fig. 4. The fibers had an oval to rectangular cross-section as seen from Fig. 5 similar to that of the common wild silks but unlike B. mori silks that have a triangular cross-section that provides excellent luster to the fibers [21]. Visually, the A. lunas fibers were dull compared to B. mori silk.

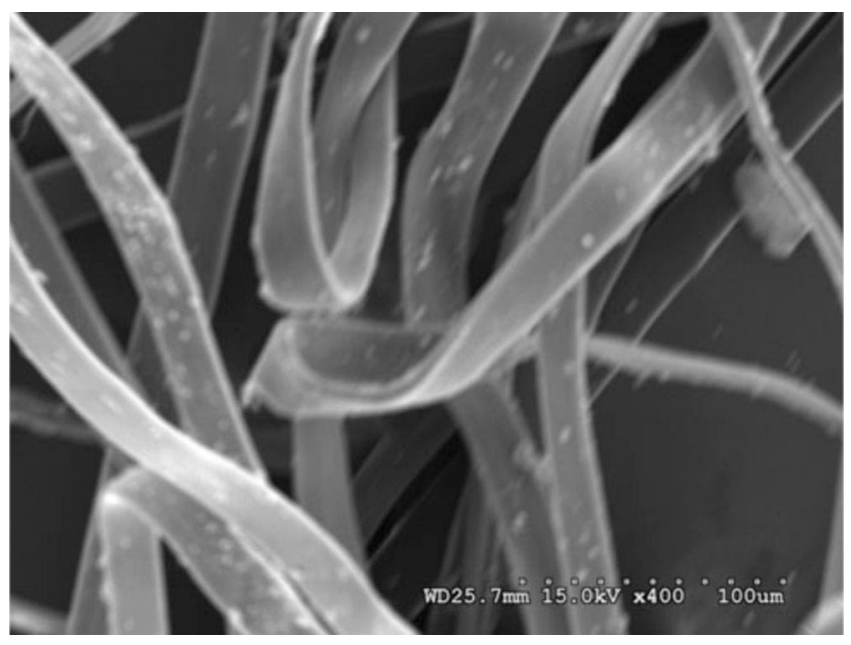

Fig. 3. SEM image revealing the flat and ribbon-like A. lunas silk fibers.

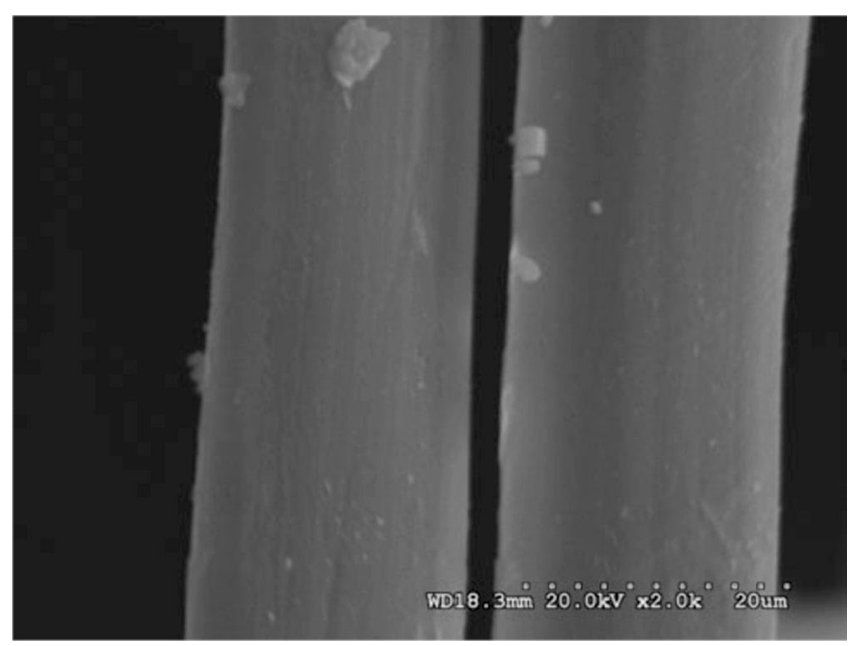

Fig. 4. SEM image of the longitudinal surface of degummed $A$. lunas silk fibers shows a clean and smooth surface.

\section{Physical Structure}

The A. lunas silk fibers had a considerably different physical structure than the $B$. mori silk fibers as seen from Fig. 6. However, the diffractogram of the A. lunas fibers was similar to that of the wild silks. The A. lunas silk had two diffracting peaks at about $16.8^{\circ}$ and $21.1^{\circ}$ corresponding to a d-spacing of 5.27 and $4.41 \AA$, respectively as seen in A. assama silk [20]. The B. mori silk had a single prominent peak at about $20.6^{\circ}$. In addition to the two prominent peaks, the A. lunas fibers had a small sharp peak at about $29^{\circ}$ which was due to the presence of the calcium oxalate crystals [20]. The \% crystallinity of the A. lunas fibers was $30.6 \%$ was in the range of $\%$ crystallinity reported for B. mori (20-41 \%) silk. Overall, the physical structure of $A$. lunas silk was found to be more close to that of the wild silk than B. mori silk. 


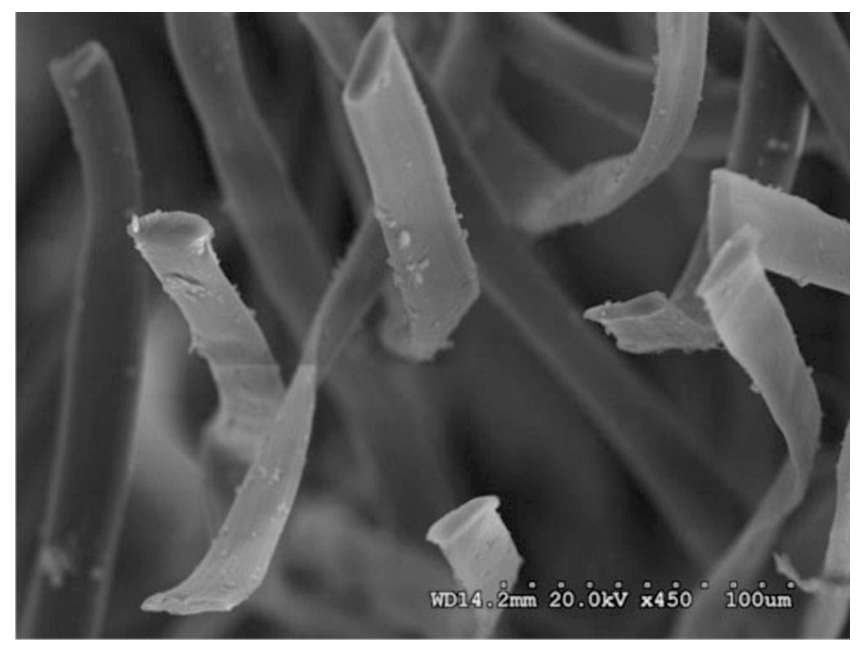

Fig. 5. SEM image of the cross-section A. lunas silk fibers shows that the fibers are solid and have oval to rectangular cross-sections.

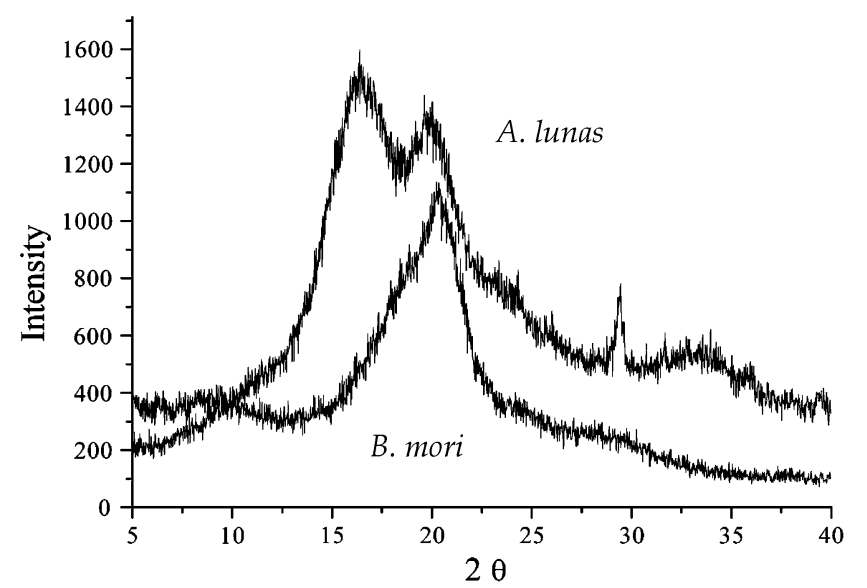

Fig. 6. X-ray diffractogram of A. lunas silk fibers compared to B. mori silk fibers.

\section{Tensile Properties}

Table 3 provides a comparison of the fineness, tensile properties and moisture regain of the A. lunas fibers with $B$. mori and two common wild silks. As seen from the table, the A. lunas fibers were coarser than B.mori but much finer than the wild silks. It is reported that the coarseness of the wild silks is a major reason for the limited applications of the wild silks compared to mulberry silk [17, 18, 21]. The much finer fibers produced by $A$. lunas compared to the wild silks suggests that the fibers could be more suitable for applications currently using B. mori silk. The breaking tenacity of the A. lunas fibers was higher than that of the wild silks but similar to that of the B. mori silk. However, the breaking elongation of the A. lunas fibers was much lower than that of the wild silks. The modulus of the fibers was lower than B. mori, similar to that of A. mylitta but higher than
Table 3. Tensile properties of A. lunas silk fibers compared to Bombyx mori, A. mylitta and $P$. ricini silks

\begin{tabular}{lllll}
\hline Fiber Property & A. lunas & B. mori & A. mylitta & P. ricini \\
\hline $\begin{array}{l}\text { Fineness, denier } \\
\text { Breaking tenacity, } \\
\text { g/den }\end{array}$ & $2.0 \pm 0.2$ & $0.4-1.1$ & $4.7-10.7$ & $2.3-3.6$ \\
$\begin{array}{c}\text { Breaking } \\
\text { elongation, \% }\end{array}$ & $10.9 \pm 4.5$ & $10.0-23.4$ & $26-39$ & $24-27$ \\
$\begin{array}{c}\text { Young's modulus, } \\
\text { g/den }\end{array}$ & $76 \pm 11$ & $84-121$ & $66-70$ & $29-31$ \\
$\begin{array}{l}\text { Moisture regain, \% } \\
\text { Moin }\end{array}$ & 12.1 & $8.5-9.9$ & 10.5 & 10.0 \\
\hline
\end{tabular}

Data for B. mori and wild silks are from references [17, 18, 22].

that of $P$. ricini silk suggesting that the $A$. lunas fibers may not be as smooth as $B$. mori but are not as harsh to handle as the wild silks. The relatively high breaking tenacity of the A. lunas fibers despite having low \% crystallinity should be due to the presence of amino acids such as trysoine that provide strong interactions. Lower elongation of A. lunas fibers compared to the wild silks should be due to the finer fibers and probably better arrangement of the protein crystals along the fiber axis. The moisture regain of the A. lunas fibers was higher than that of the other fibers in Table 3 despite the fibers having higher amounts of hydrophobic amino acids than the common silks. This should be due to the differences in the arrangement of the amino acids in the fibers and the \% crystallinity.

\section{Conclusions}

Actias lunas produced cocoons that were considerably smaller and lighter that the commonly known silks. Fibers obtained after degumming the cocoons had much lower amount of glycine but higher amounts of tyrosine, aspartic acid, arginine and leucine compared to B. mori and the common wild silks. The A. lunas fibers had lower ratio of crystalline to amorphous amino acids but had higher amounts of hydrophobic amino acids. Physical structure of the A. lunas fibers was more similar to that of the wild silks than B. mori silk. Morphologically, the fibers were flat and ribbon-like with a oval to rectangular cross-section that makes the fibers less lustrous compared to B. mori silk fibers. The A. lunas fibers are coarser than the $B$. mori silk but much finer than the wild silks. However, the breaking tenacity of the luna fibers was similar to that of $B$. mori fibers but higher than that of the wild silks. The breaking elongation of the luna fibers was similar to that of $B$. mori silk but lower than that of the wild silks. Overall, the A. lunas silk fibers show potential to overcome the limitations of wild silks and be useful for applications that currently use mulberry silks. 
Acknowledgments - The authors wish to thank the Agricultural Research Division at the University of Nebraska-Lincoln, USDA Hatch Act, and Multi-state Project S1026 for their financial support to complete this research.

\section{References}

1. Engelhardt A (2010) Fiber year 2008-2009: a world survey on textile and non-woven industry. Saurer, Remscheid, Germany

2. Reddy N, Yang Y (2007) Biomacromolecules 8(2):638

3. Reddy N, Yang Y (2008) J Mater Sci Mater Med 19:2055

4. Reddy N, Yang Y (2009) Biotechnol Prog 25(6):1796

5. Tuskes PM, Tuttle JP, Collins MM (1996) The wild silk moths of North America: the natural History of the Saturniidae of the United States and Canada. Cornell University Press, Ithaca

6. Brown SA, Ruxton GD, Humphries S (2004) J N Am Benthol Soc 23(4):771

7. Sutherland TD, Weisman S, Trueman HE, Sriskantha A, Trueman JWH, Haritos VS (2007) Mol Biol Evol 24(11):2424

8. Weisman S, Trueman HE, Mudie ST, Church JS, Sutherland TD, Haritos VS (2008) Biomacromolecules 9(11):3065
9. Razafimanantosoa T, Ravoahangimalala OR, Craig CL (2006) Madag Conserv Dev 1(1):34

10. Nurmalitasari G, Kuroda F (2002) Fourthth international conference on wild silkmoths, 23-26 April, Yogyakarta, Indonesia

11. Siri S, Maensiri S (2010) Int J Biol Macromol 46:529

12. Kasoju N, Bhonde RR, Bora U (2009) Mater Lett 63(28):2466

13. Reddy N, Yang Y (2010) Int J Biol Macromol 46(4):419

14. Reddy N, Yang Y (2010) J Mater Sci 45:4414

15. Ferguson DC (1972) The moths of America North of Mexico. In: Domino RB, Edwards CR, eds., Classey Ltd, Middlesex, England, pp 41-56

16. Saha M, Mahendran B, Kundu SC (2008) J Econ Entomol 101(4):1176

17. Sen K, Babu MK (2004) J Appl Polym Sci 92:1080

18. Sen K, Babu MK (2004) J Appl Polym Sci 92:1098

19. Trovelot L (1867) Am Nat 1(2):85

20. Freddi G, Gotoh Y, Mori T, Tsutsui I, Tsukada M (1994) J Appl Polym Sci 52:775

21. Robson RM (1998) Handbook of fiber chemistry. In: Morton WE, Hearle JWS (eds) Marcel Dekker Inc., New York, pp 415-429

22. Saravanan D (2006) J Text Appar Technol Manag 5(1):1 


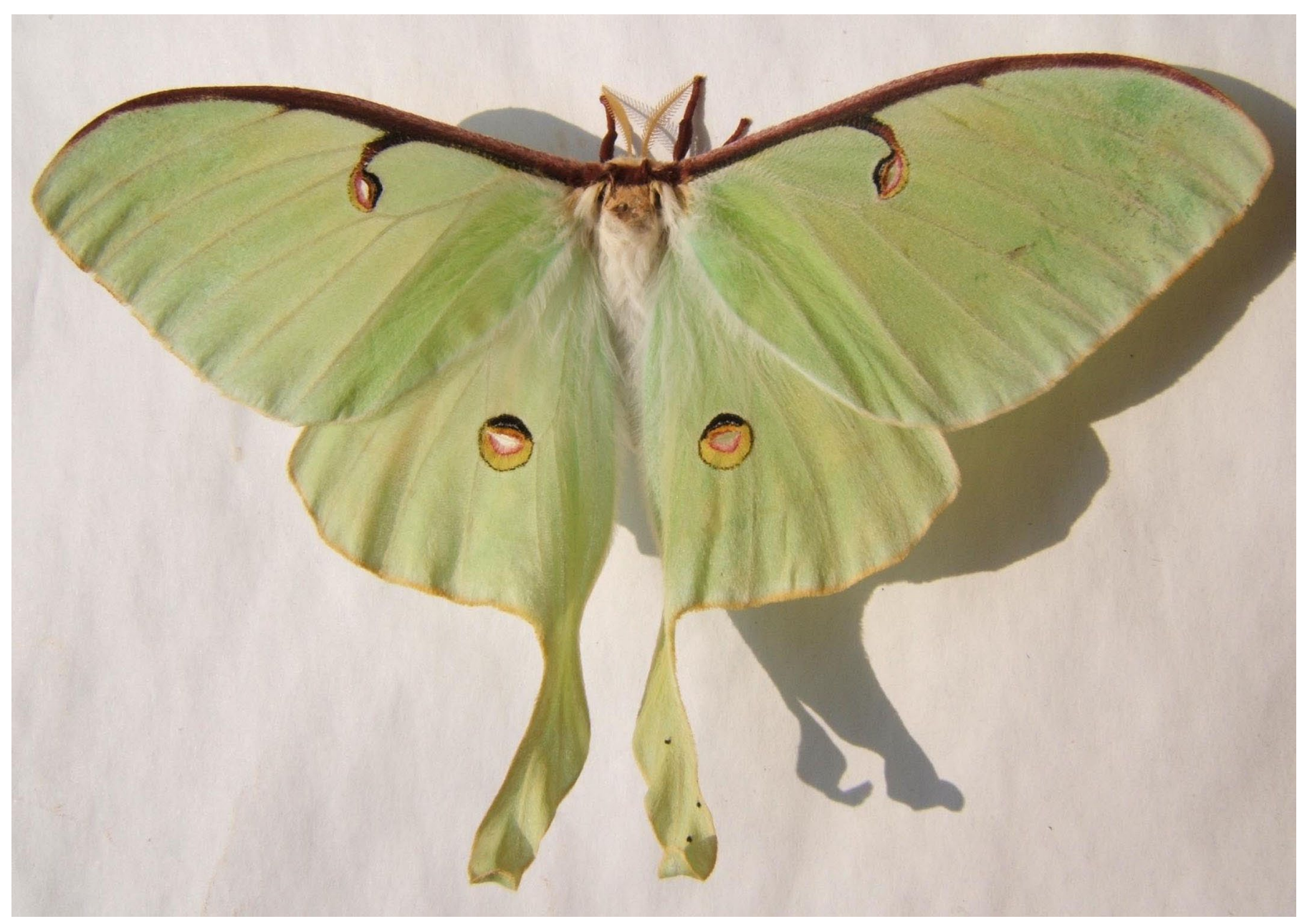

Actias luna female taken by Shawn Hanrahan and reared on American sweetgum; http://en.wikipedia.org/wiki/File:Actias luna female sjh.JPG

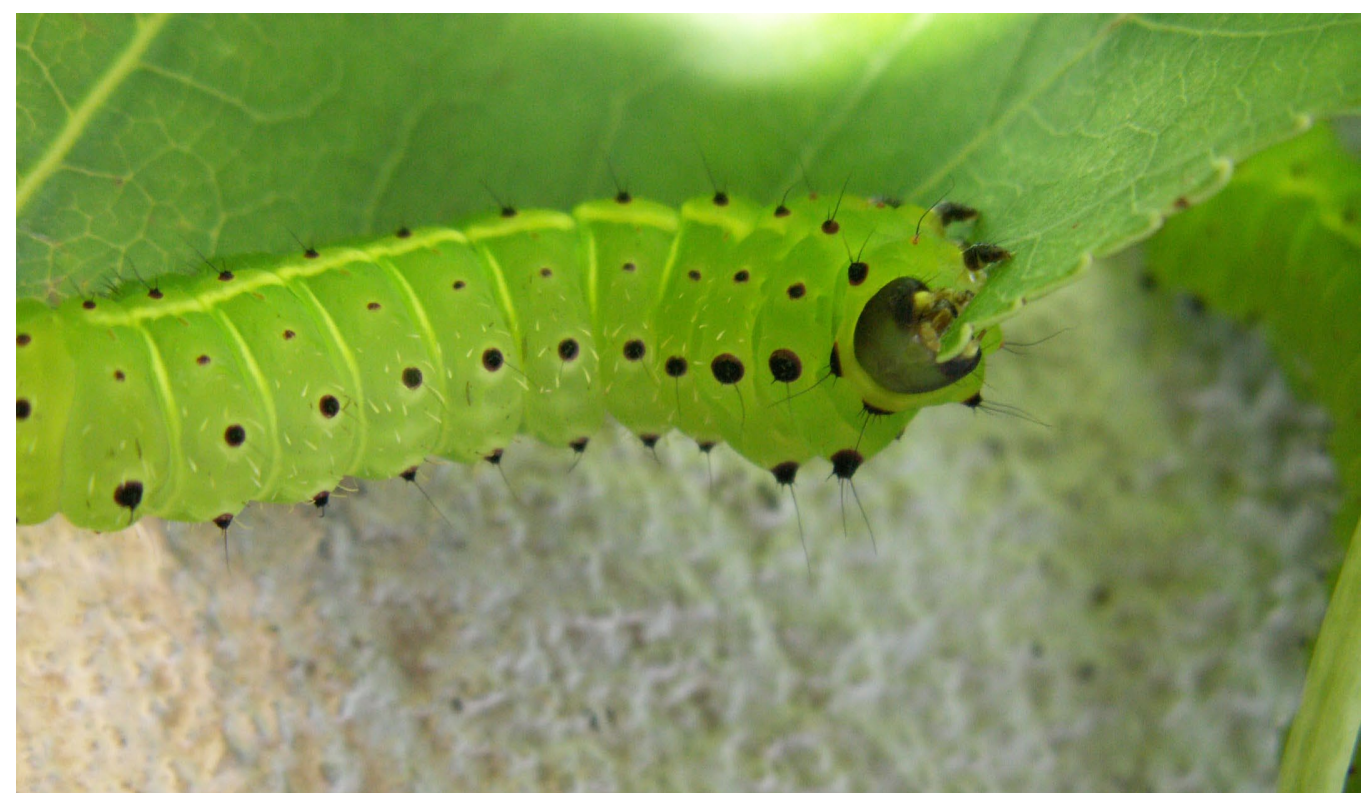

Actias luna 4th instar larva taken by Shawn Hanrahan. Reared on American sweetgum; http://en.wikipedia.org/wiki/File:Actias_luna_4th_instar_sjh.JPG 